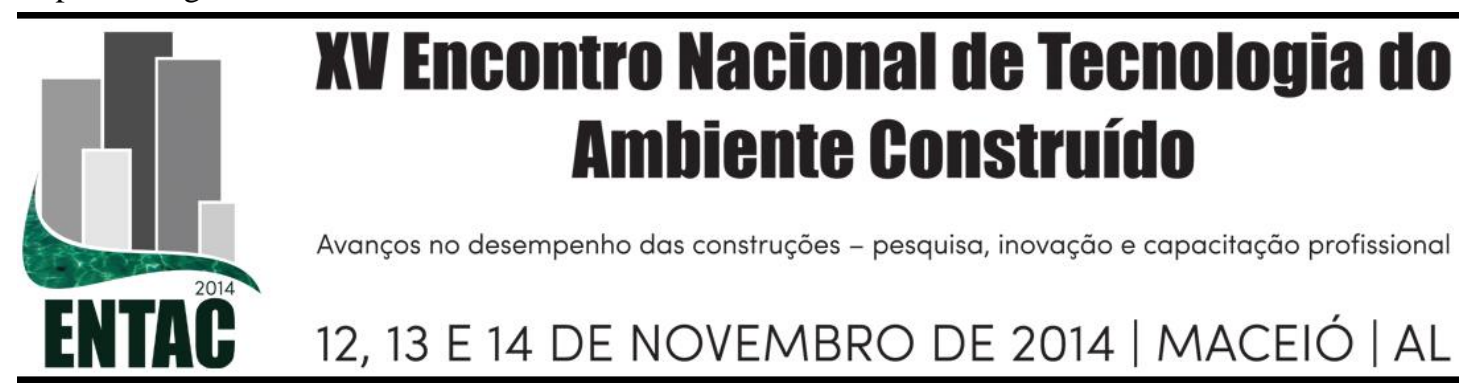

\title{
INSERÇÃO E FINANCIAMENTO DO SISTEMA WOOD FRAME NO PROGRAMA HABITACIONAL MINHA CASA MINHA VIDA
}

\author{
ESPINDOLA, Luciana da Rosa (1); INO, Akemi (2)
}

(1) Instituto Federal de Educação, Ciência e Tecnologia de Santa Catarina, luciana.espindola@ifsc.edu.br (2) Instituto de Arquitetura e Urbanismo - Universidade de São Paulo, inoakemi@ usp.sc.br

\begin{abstract}
RESUMO
No Brasil, em geral, as inovações tecnológicas para construções em madeira foram caracterizadas por eventos pontuais. Entre os diversos entraves para este avanço tecnológico destaca-se a dificuldade de financiamento das construções em madeira por agentes nacionais. No entanto, recentemente, após a instituição do Sistema Nacional de Avaliação Técnica (SINAT), o qual possibilita o financiamento de construções com tecnologias ainda não normatizadas no Brasil, um grupo atuante na indústria da construção em madeira deliberou parâmetros normativos específicos para construções em Wood Frame. Como consequência, mediante o programa habitacional Minha Casa Minha Vida (MCMV) em parceria com a Caixa Econômica Federal (CEF), em meados de 2012, foi executado o primeiro conjunto habitacional em Wood Frame no Brasil - o residencial Haragano. Portanto, sob este novo contexto, o presente trabalho tem como objetivo analisar tal inserção e o financiamento do sistema construtivo Wood Frame no programa MCMV. Para isto, o método deste trabalho é elaborar uma análise crítica teórica sobre os seguintes temas principais: condicionantes gerais para o financiamento de habitações em madeira; processo de regulamentação normativa para o financiamento do Wood Frame no contexto brasileiro; e, por fim, implementação do conjunto habitacional Haragano. Os dados apresentados constituem uma pesquisa de doutorado em andamento. Os resultados parciais indicam que o posicionamento de restrição da $\mathrm{CEF}$ às edificações em madeira está diretamente atrelado ao baixo desempenho em geral desta oferta. No entanto, após publicação da diretriz SINAT número 005 específica para construções em Wood Frame, a CEF possibilitou o financiamento do primeiro conjunto habitacional com este sistema construtivo. Com base nesta execução e nos ensaios realizados por Instituições Técnicas Avaliadoras (ITA’s), a CEF está propensa a financiar novos conjuntos habitacionais em madeira. Portanto, conforme estes avanços ocorrem, as fragilidades devem ser identificadas para não degradar as vantagens consolidadas destas construções em madeira.
\end{abstract}

Palavras-chave: Construção em madeira, Financiamento, Programa habitacional.

\begin{abstract}
Generally in Brazil, wood constructions innovations are remarked by punctual events. Among the obstacles to improve this sector is the difficulty of financing wooden constructions by national agents. However, recently, after establishing the National System for Technical Evaluation (SINAT) that allows financing constructions built with technologies not yet standardized in Brazil, a group of the wood construction industry deliberated standards for Brazilian wood frame buildings. Consequently, in 2012, the first social housing development named Haragano was implemented in the program Minha Casa Minha Vida (MCMV) financed by Caixa Econômica Federal (CEF). Therefore, in this new context, the present paper aims to analyze the inclusion and the financing of wooden frame construction in the housing program MCMV. For this, the method of this work is to develop a theoretical investigation on the following main themes: general conditions for financing wooden buildings; the developing process of standards for wood frame constructions in Brazil; and, at last, the implementation of Haragano's social housing development. The data presented are from a PhD research in progress. The partial results indicate that the CEF's financing restriction to wooden buildings is directly linked to theirs low performance in general. However, after the publication of the guideline SINAT number 005 for Brazilian
\end{abstract}


Wood Frame buildings, CEF financed the first social housing development applying this specific construction technique. Based on this implementation and on the tests evaluated by Technical Institutions (ITA's), CEF is now predisposed to finance other housing developments in wood. Therefore, as these advances occur, the possible flaws should be identified in order to maintain these wooden buildings advantages.

Keywords: Wood frame, Financing, Social housing program.

\section{INTRODUÇÃO}

No Brasil, em geral, as inovações tecnológicas para construções em madeira foram caracterizadas por eventos pontuais. Após a importante atuação dos imigrantes europeus, as técnicas construtivas em madeira sofreram poucas alterações significativas. No decorrer histórico, há referências que demonstram as boas práticas e as distintas possibilidades das estruturas em madeira. No entanto, apesar das vantagens comprovadas, a madeira não é considerada um material convencional no contexto brasileiro. Execuções errôneas denegriram e sobrepuseram as vantagens deste material. Gradativamente, o conhecimento prático dos carpinteiros pioneiros foi perdido e, consequentemente, desacertos nas construções em madeira acarretaram na falta de qualidade das mesmas, assim preconizando este conceito na população.

Associadas ao conceito popular negativo, as decorrentes restrições para o financiamento das edificações em madeira mediante agentes nacionais também balizaram o avanço tecnológico deste setor no Brasil. Para viabilizar um financiamento, além dos critérios de viabilidade econômica, as normas de desempenho técnico da construção devem ser atendidas. Portanto, a generalização da falta de qualidade apresentada pelas construções em madeira vem contribuindo para o indeferimento destes financiamentos.

Visando transformar este cenário, em 2009, um grupo atuante na indústria da construção em madeira organizou-se na denominada Comissão Casa Inteligente com intuito de definir diretrizes de desempenho para o sistema construtivo Wood Frame, ainda não consolidado no Brasil. Esta atuação foi motivada pela instituição do Sistema Nacional de Avaliação Técnica (SINAT) no âmbito do Programa Brasileiro da Qualidade e Produtividade do Habitat (PBQP-H) através do Ministério das Cidades em 2007. Pois, o estabelecimento de diretrizes SINAT e as comprovações técnicas para sistemas que não possuem normas brasileiras específicas possibilitam que os mesmos sejam financiados por agentes como a Caixa Econômica Federal (CEF) e participem nos programas habitacionais vigentes.

Seguindo este processo, em 2011, é aprovada a Diretriz SINAT $\mathrm{n}^{\circ} 005$ intitulada "Sistemas construtivos estruturados em peças de madeira maciça serrada, com fechamentos em chapas delgadas - Sistemas leves tipo Light Wood Framing" (BRASIL, 2011). Em sequência, em meados de 2012, a CEF aprova a construção do primeiro conjunto habitacional em Wood Frame, incluso no programa Minha Casa Minha Vida (MCMV) - o residencial Haragano, localizado na cidade de Pelotas, Rio Grande do Sul.

Tendo em vista esta transformação no contexto do setor da construção em madeira no Brasil, o presente trabalho tem como objetivo analisar a inserção e o financiamento do sistema construtivo Wood Frame no programa MCMV. Para isto, o método deste trabalho é elaborar uma análise crítica teórica sobre os seguintes temas principais: condicionantes gerais para o financiamento de habitações em madeira; processo de regulamentação normativa para o financiamento do Wood Frame no contexto brasileiro; e, por fim, implementação do conjunto habitacional Haragano. Os dados apresentados a 
seguir constituem uma pesquisa de doutorado em andamento e foram obtidos através de levantamentos de campo, incluindo entrevistas.

\section{CONDIÇÕES DE FINANCIAMENTO PARA HABITAÇÃO EM MADEIRA}

A Caixa Econômica Federal (CEF) é atualmente a principal instituição financiadora no Brasil, atuando como banco público "responsável pela operação das políticas públicas do Governo Federal voltadas para habitação e cidades" (BUENO, 2008, p. 22). Na operacionalização do seu papel, a CEF exige que a unidade habitacional atenda aos critérios de desempenho da NBR 15575 (2013) e do PBQP-H. Além destas normativas, a CEF apresenta parâmetros próprios para a viabilização do financiamento de habitações para sistemas construtivos convencionais e inovadores.

Para o financiamento de construções convencionais em madeira, a CEF apresenta os critérios descritos na tabela 1, apresentados pela Gerência de Desenvolvimento Urbano e Rural de Porto Alegre (GIDUR/PO) em entrevista realizada em 2012.

Tabela 1 - Orientações para financiamento de habitações em madeira da CEF

\begin{tabular}{|l|c|c|c|}
\hline \multicolumn{1}{|c|}{ Casas térreas e sobrados } & A construir & Novo & Usado \\
\hline Prazo máximo & 15 anos & 15 anos & 15 anos \\
\hline $\begin{array}{l}\text { Valor limite para financiamento } \\
\text { (com áreas molháveis em alvenaria) }\end{array}$ & $100 \%$ & $100 \%$ & $100 \%$ \\
\hline $\begin{array}{l}\text { Valor limite para financiamento } \\
\text { (sem áreas molháveis em alvenaria) }\end{array}$ & $80 \%$ & $80 \%$ & $80 \%$ \\
\hline Termo de ciência & Sim & Sim & Sim \\
\hline Termo de responsabilidade do responsável técnico & Sim & Sim & Sim \\
\hline Termo do fornecedor & Sim* & Sim* & Não \\
\hline Vistoria sem patologia CAIXA & Não & Sim & Sim \\
\hline ART de cálculo estrutural & Não & Sim & Sim \\
\hline $\begin{array}{l}\text { Comprovação de desempenho (Relatório Técnico } \\
\text { de Avaliação - RTA) }\end{array}$ & Não & Não & Não \\
\hline Manual do proprietário - manutenção & Sim & Sim & Não \\
\hline *Comprovação de utilização de madeira extraída de floresta com manejo controlado. \\
\hline
\end{tabular}

Fonte: ELTZ, SPEGGIORIN (2012)

Apesar destas orientações gerais estabelecidas para todo território nacional, segundo Eltz e Speggiorin (2012), os procedimentos para o financiamento de habitações em madeira pela CEF "variam entre os diversos estados, tendo em vista aspectos locais, culturais, tecnológicos, de logística e de mercado". Por exemplo, no estado do Rio Grande do Sul, para garantir que o produto final apresente melhor desempenho e maior durabilidade, o financiamento é restrito às unidades individuais respeitando os seguintes apontamentos:

- Comprovação legal da origem da madeira, indicando os procedimentos de secagem, desdobramento, beneficiamento e imunização da mesma;

- Especificação da espécie de madeira e dos tipos de tratamento e de imunização aplicados;

- Utilização da espécie Pinus elliotii restrita aos elementos de forros e de divisórias internas; 
- Utilização de espécies de madeira de alta densidade para assoalho, esquadrias e paredes externas, incluindo lambris;

- Execução obrigatória de paredes externas duplas, inserindo material isolante no interior destas a fim de garantir seu desempenho térmico e acústico - exceto nos casos de paredes compostas por peças com espessura de 02 polegadas ou 5 $\mathrm{cm}$, aproximadamente;

- Proteção efetiva à penetração de umidade nas paredes externas utilizando produtos específicos para esta função;

- Execução da cobertura com estrutura reforçada, beirais avantajados e fechamento com telhas cerâmicas ou de concreto.

Observa-se, dentre estas condicionantes, a questão da durabilidade atrelada à seleção da espécie de madeira utilizada. Para os principais elementos da edificação, especialmente os expostos às intempéries, priorizam-se espécies densas, popularmente consideradas mais nobres. O receio quanto à aplicação de espécies de baixa densidade, como o caso específico do pinus, refere-se a sua durabilidade e suscetibilidade à ação de insetos xilófagos. Portanto, faz-se necessária a comprovação de tratamento para imunização destas peças.

Ainda, outro aspecto apontado é a qualidade da edificação condicionada ao processo de produção dos elementos em madeira. Usualmente, esta produção é diretamente dependente de fornecedores responsáveis pelos procedimentos de secagem, desdobramento e beneficiamento da matéria-prima. Assim sendo, estas etapas devem ser fiscalizadas e registradas para o controle de qualidade da madeira fornecida. Porém, constatam-se problemas frequentes com o fornecimento das peças de madeira, sendo estas apresentadas com umidade indevida, defeitos ou falta de padronização nas dimensões das seções.

Em levantamentos realizados em três empresas construtoras da região sul, as quais aplicam o sistema Wood Frame com componentes pré-fabricados, estimou-se aproximadamente $30 \%$ de perda das peças fornecidas de pinus, principalmente por apresentarem defeitos como presença de nós e variações de curvatura. Apesar das possibilidades de reutilização destas perdas para elaborar componentes complementares, ressalta-se a baixa qualidade da madeira ofertada para o setor da construção, com destaque às espécies oriundas de florestas plantadas.

Dessa forma, conforme a GIDUR/PO da CEF, em geral, as construções em madeira no país não atendem os critérios estabelecidos de desempenho e são ofertadas com baixa qualidade. Consequentemente, o financiamento dessas habitações é frequentemente inviabilizado, como descrito na entrevista realizada:

\footnotetext{
Entendemos que, em princípio, a oferta de edificações em madeira no mercado da construção civil tem se caracterizado por produtos com baixa tecnologia, ausência de controle de qualidade dos diversos componentes, ausência de certificação de origem da madeira e processo de beneficiamento deficiente, gerando edificações de baixo desempenho e durabilidade, salvo algumas exceções, o que explica a postura conservadora da CAIXA em relação a estas tipologias (ELTZ, SPEGGIORIN, 2012).
}

As deficiências na etapa de produção dos componentes somadas ao desconhecimento sobre as especificidades da madeira majoram as edificações ofertadas com baixa qualidade. 
Com intuito de reverter a tal situação presente, desde meados de 2009, empresas do setor madeireiro reuniram-se com pesquisadores, construtores, fornecedores $\mathrm{e}$ consultores para estabelecer critérios normativos de desempenho e qualificar as construções em Wood Frame, a fim de possibilitar financiamento das mesmas pela CEF.

\section{REGULAMENTAÇÃO PARA O FINANCIAMENTO DO WOOD FRAME}

Desde meados da década de 1990, os sistemas construtivos leves em madeira, entre estes o Wood Frame, são contemplados no Brasil por pesquisas acadêmicas que analisaram suas possibilidades de adaptação e inserção no contexto brasileiro. Apesar dos resultados positivos obtidos, a propagação destes sistemas ocorreu em pequena escala no mercado.

Em junho de 2009, uma comitiva formada por empresários e engenheiros brasileiros visitou fábricas localizadas na Alemanha para compreender o processo de produção industrializada do sistema Wood Frame. Assim, instituiu-se uma parceria entre o Sistema Nacional de Aprendizagem Industrial do Paraná (SENAI-PR) e o Ministério das Finanças e da Economia do estado alemão Baden-Württemberg para possibilitar a transferência destas tecnologias ao Brasil (TÉCHNE, 2009).

No Brasil, este grupo convocou uma reunião com interessados em elaborar uma regulamentação específica para o Wood Frame no Brasil, com objetivo de contemplá-lo nas agências de financiamento nacional. (BUETTNER, 2009).

Em novembro de 2009, foi constituída oficialmente a Comissão Casa Inteligente pertencente ao Conselho Setorial da Indústria de Base Florestal da Federação das Indústrias do Estado do Paraná (FIEP), a qual elaborou um modelo de diretrizes técnicas para o Wood Frame no Brasil (FIEP, 2009).

Em setembro de 2011, as diretrizes propostas por esta comissão foram aprovadas, resultando no documento intitulado "Diretriz $\mathrm{n}^{\circ}$ 005: Sistemas construtivos estruturados em peças de madeira maciça serrada, com fechamentos em chapas delgadas - Sistemas leves tipo Light Wood Framing" (BRASIL, 2011).

\subsection{Diretriz SINAT $n^{\circ} 005$}

Em 2007, precedendo o lançamento do programa habitacional MCMV, é instituído o Sistema Nacional de Avaliação Técnica (SINAT) vinculado à Secretaria Nacional de Habitação do Ministério das Cidades. O SINAT, baseado no conceito de desempenho do PBQP-H, cria diretrizes técnicas para produtos inovadores da construção civil, ou seja, sistemas ou subsistemas que não possuem norma técnica brasileira para análise de desempenho. Esta é uma mudança significativa que possibilita o financiamento pela CEF de construções com inovações tecnológicas (BRASIL, 2007).

Nesse quadro, a diretriz SINAT $n^{\circ} 005$ define parâmetros para os componentes de piso, parede e cobertura do sistema construtivo estruturado por peças de madeira maciça serrada com fechamento por chapas delgadas, definido Light Wood Frame. Esta diretriz limita-se às unidades habitacionais unifamiliares do tipo térreo ou sobrado, isoladas ou geminadas (BRASIL, 2011).

Haja vista a preocupação frequente referente à durabilidade das peças de madeira, a diretriz 005 elencou critérios detalhados neste sentido. A madeira empregada deve ser de origem legal comprovada. As peças estruturais devem ser tratadas quimicamente para minimizar o ataque de organismos xilófagos. Além do tratamento, solicita-se um 
detalhamento específico no projeto para evitar o contato ou a permanência da umidade nestas peças.

De forma geral, seguindo a NBR 15575 (2013), os seguintes requisitos de desempenho devem ser atendidos: resistência estrutural, segurança contra incêndio, estanqueidade à água, desempenho térmico, desempenho acústico, durabilidade e manutenabilidade.

Todavia, o desempenho global da edificação também está relacionado ao controle da qualidade na fase de montagem da mesma. No caso das construções em Wood Frame, as etapas de montagem podem ocorrer no canteiro de obras, nas unidades industriais ou em parcialmente em ambos. Neste aspecto, segundo a diretriz SINAT 005 (BRASIL, 2011), o construtor deve comprovar o controle de aceitação de materiais, bem como garantir a rastreabilidade das informações.

Por fim, após a execução da edificação, é imprescindível uma análise geral para identificar, avaliar e corrigir a existência de não conformidades que possam causar prejuízos ao desempenho do sistema (BRASIL, 2011).

A proposta destas diretrizes aprovadas pelo SINAT foi o primeiro procedimento para possibilitar o financiamento de construções em Wood Frame no Brasil pela CEF. Na sequência, o sistema construtivo proposto, incluso seus componentes constituintes, devem ser testados segundo as normas técnicas pertinentes para que o SINAT conceda o Documento de Avaliação Técnica (DATec).

\subsection{DATec $\mathbf{n}^{\circ} \mathbf{0 2 0}$}

O pedido de um DATec deve ser realizado por um proponente, o qual será seu proprietário após a concessão final. Para receber um DATEc, o proponente demonstra os detalhes técnicos de composição, execução e manutenção do sistema construído seguindo as instruções da diretriz SINAT relacionada. Estes elementos construtivos são testados por Instituições Técnicas Avaliadoras (ITA), as quais transcrevem os resultados nos Relatórios Técnicos de Avaliação (RTA). Após a análise e a aprovação dos dados contidos no RTA por uma auditoria técnica, o DATec é concedido (BRASIL, 2007).

No caso específico do Wood Frame, o pedido do DATec foi realizado pela Tecverde Engenharia LTDA. Os diversos ensaios de desempenho foram realizados por diferentes ITA's. No entanto, o Instituto Falcão Bauer da Qualidade (IFBQ), ITA coordenadora deste processo, assegurou o desempenho do sistema construtivo no RTA final (BRASIL, 2013).

Após apreciação dos resultados, em outubro de 2013, a Comissão Nacional do SINAT concedeu ao "Sistema construtivo TECVERDE: sistema leve em madeira" o DATec ${ }^{\circ}$ 20, restrito à construção de unidades habitacionais unifamiliares térreas isoladas ou geminadas. O documento descreve o sistema construtivo em questão, conforme ilustrado na Figura 1.

As paredes externas e internas, com função estrutural, são formadas por quadros estruturais em peças de madeira serradas autoclavadas. O fechamento da face externa das paredes de fachada é realizado em chapas de OSB com função de contraventamento, revestidas de placas cimentícias com tratamento de juntas aparentes. $\mathrm{O}$ fechamento da face interna das paredes de fachada e de ambas as faces das paredes internas é realizado em chapas de gesso acartonado para drywall. As paredes de geminação possuem núcleo em manta de lã de vidro. A cobertura é constituída de estrutura metálica, telhado em telhas cerâmicas, forro em réguas de PVC e manta de lã de rocha posicionada sobre o forro (BRASIL, 2013, p. 2). 


\section{Figura 1 - Composição do elemento de parede do sistema Tecverde}

\begin{tabular}{l}
1 Estrutura de madeira \\
autoclavada de \\
alta densidade \\
2 Isolante térmico \\
e acústico \\
3 Placa de OSB \\
\hline 4 Membrana hidrófuga \\
\hline 5 Placa cimentícia \\
(do lado externo) \\
6 Placa de gesso \\
acartonado (do lado interno) \\
7 Acabamento
\end{tabular}

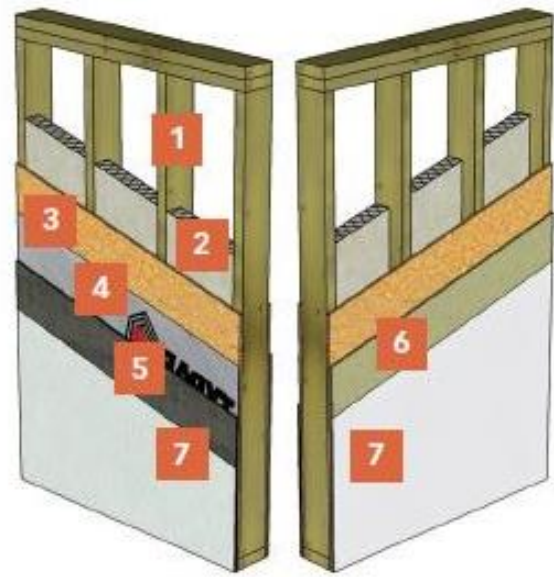

Fonte: Ferreira (2013)

A empresa Tecverde é a detentora do DATec $\mathrm{n}^{\circ} 20$ e, consequentemente, desta tecnologia proposta para construção em Wood Frame. Assim, a mesma está habilitada a pleitear o financiamento destas habitações pela CEF. Outras empresas que desejam obter o financiamento da CEF utilizando esta proposta dependem de permissão e parceria com a Tecverde, pois esta é a responsável pela qualidade da oferta deste sistema construtivo quando associado ao DATec $\mathrm{n}^{\circ} 20$.

Antes da publicação oficial deste DATec, em meados de 2012, a primeira parceria realizada ocorreu entre as empresas Tecverde e Roberto Ferreira para a execução do residencial Haragano - primeiro conjunto habitacional em Wood Frame financiado pela CEF no programa MCMV.

\section{CONJUNTO HABITACIONAL HARAGANO}

O conjunto Haragano, localizado na cidade de Pelotas, no estado do Rio Grande do Sul, empreendimento da construtora Roberto Ferreira, atende a primeira faixa do PMCMV, a qual inclui famílias com renda mensal de até três salários mínimos. Construído entre os anos de 2012 e 2013 em um lote com 3,98 ha de área, compreende 280 unidades habitacionais de $45 \mathrm{~m}^{2}$, incluindo 270 sobrados geminados e dez casas térreas (FERREIRA, 2013).

A execução dos componentes constituintes ocorreu parcialmente entre unidade industrial e canteiro de obras, sendo assessorado em todas as etapas pela construtora Tecverde.

Os painéis de parede e piso foram pré-fabricados seguindo os critérios da diretriz SINAT 005. Na fábrica, também localizada em Pelotas, uma linha de montagem subdividida em estações de trabalho era composta por ferramentas e maquinários específicos manuseados por uma equipe de 18 trabalhadores, incluindo homens e mulheres. No recebimento das matérias-primas, estas eram inspecionadas, selecionadas e registradas para futuro rastreamento. Durante a confecção dos componentes, após a conclusão de cada etapa, um fichamento é realizado, pontuando os dados sobre a qualidade da execução do produto. Por fim, cada componente finalizado em fábrica é inspecionado pelo engenheiro responsável pela produção (Figura 2). 
Figura 2 - Pré-fabricação dos painéis de parede e piso do conjunto Haragano

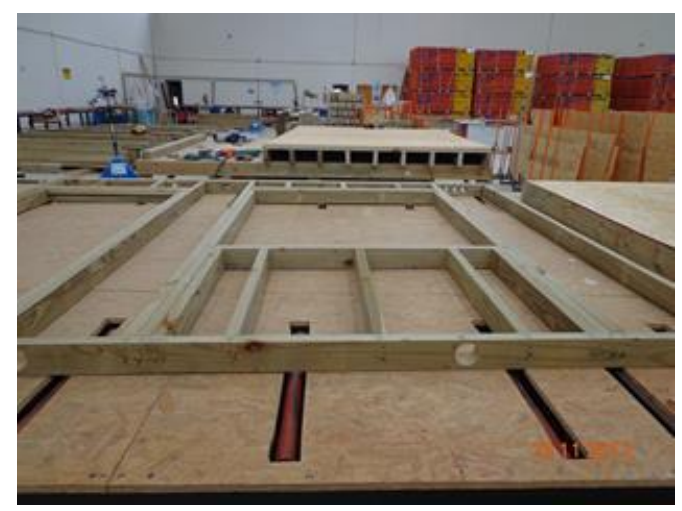

Fonte: Autor (2012).

Após a pré-fabricação, os painéis de piso e parede são transportados ao canteiro de obras, onde são montados sobre uma fundação radier. $O$ controle da montagem é realizado por um engenheiro específico que acompanha a obra, conferindo o posicionamento de cada componente pré-fabricado para manter a rastreabilidade dos elementos constituintes. Com a montagem concluída, a execução das esquadrias, das escadas, das instalações elétricas e hidrossanitárias e dos acabamentos são realizados no canteiro (Figura 3).

Figura 3 - Montagem dos painéis de parede e piso no canteiro de obras

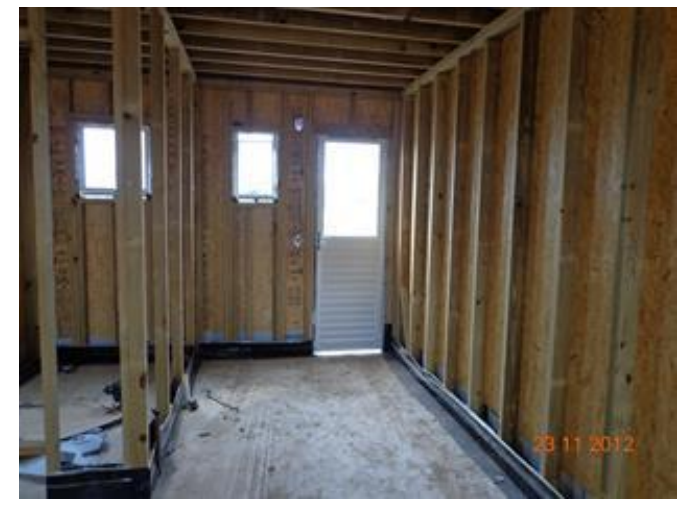

Fonte: Autor (2012).

Durante a execução do conjunto habitacional, a CEF acompanhou os relatórios mensais de monitoramento e controle tecnológico elaborados pela construtora Roberto Ferreira (ELTZ, SPEGGIORIN, 2012). Mediante os resultados apresentados, a postura positiva da GIDUR/PO da CEF sobre o sistema construtivo Wood Frame fundamenta-se na gestão da produção dos componentes pré-fabricados e da montagem no canteiro de obras, incluindo o controle da qualidade em todo o processo (BRASIL ENGENHARIA, 2013). Apesar do significante marco na aceitação da CEF, cabe a ressalva que cada solicitação de financiamento é analisada de forma específica, dependendo das propostas e dos contextos envolvidos.

Portanto, visando expandir a disseminação do sistema construtivo Wood Frame, em 2012, as empresas Tecverde e Roberto Ferreira formaram a rede iVerde, com objetivo 
de assessorar e monitorar tecnicamente outras empresas interessadas em executar o sistema (TECVERDE, 2013).

Todavia, como o DATec $n^{\circ} 020$ é restrito ao proponente, outras construtoras que utilizam o sistema Wood Frame, porém não estão interessadas na parceria com a rede, seguem caminhos paralelos para obter outras certificações para comprovar a qualidade e o desempenho de suas propostas.

Neste atual contexto, o Wood Frame tem demonstrado suas vantagens de aplicação nas regiões sul e sudeste do Brasil. De forma geral, boas práticas estão impulsionando o mercado, demonstrando o incremento da produção em fábrica e no canteiro. Porém, nesta recente euforia de transformação tecnológica, as possíveis fragilidades da cadeia de produção das construções em madeira deixam de ser expostas. Estas devem ser apontadas para evitar denegrir o sistema e manter o ritmo de inclusão, em especial para habitações sociais.

\section{CONSIDERAÇÕES FINAIS}

Este artigo apontou o motivo da postura conservadora da CEF frente ao financiamento das construções em madeira em geral. Segundo a mesma, este obstáculo é imposto pela qualidade insuficiente destas edificações em geral. Sem o conhecimento técnico e a habilidade prática, produtos são ofertados com erros de projeto e execução, gerando edificações com baixo desempenho e durabilidade.

Recentemente, para transformar este setor, um grupo denominado Comissão Casa Inteligente engajou-se para elaborar parâmetros normativos com intuito de introduzir o sistema construtivo Wood Frame no contexto brasileiro de forma significativa. Como consequência, em 2011, foi publicada a diretriz SINAT 005, especificando requisitos de desempenho para os sistemas construtivos leves em madeira. Tal diretriz constituiu a primeira transformação no cenário, criando a possibilidade para o financiamento destas construções.

No entanto, a viabilidade do financiamento do sistema Wood Frame mediante a CEF apenas se tornou possível com a concessão do DATec $n^{\circ} 020$, proposto pela empresa Tecverde. Esta empresa, detentora deste documento, realizou uma parceria com a construtora Roberto Ferreira para executar o primeiro conjunto habitacional em Wood Frame, financiado pela CEF, inserido no programa MCMV.

Com base na gestão e no controle de qualidade apresentados desde a produção dos componentes pré-fabricados até a montagem no canteiro de obras do conjunto Haragano, a CEF alterou sua postura frente às construções em madeira. No entanto, apesar dos resultados positivos, as novas solicitações de financiamento devem evidenciar o cumprimento da diretriz SINAT 005 ao apresentar edificações com desempenhos adequados.

Em paralelo, outras empresas construtoras interessadas em financiar aplicando o sistema Wood Frame, porém as quais não pretendem estabelecer parcerias com o DATec $\mathrm{n}^{\circ} 020$, buscam outras alternativas de certificação de desempenho dos seus produtos.

Nesse novo ímpeto pelo avanço tecnológico do setor, as possíveis fragilidades da cadeia de produção das construções em madeira devem ser expostas para a busca das possíveis soluções, assim evitando denegrir a imagem deste consolidado sistema neste novo contexto. 


\section{AGRADECIMENTOS}

Ao Instituto Federal de Educação, Ciência e Tecnologia de Santa Catarina (IFSC Florianópolis), ao PPGAU do Instituto de Arquitetura e Urbanismo (IAU USP - São Carlos) e à Coordenação de Aperfeiçoamento Pessoal de Ensino Superior (CAPES), pelo apoio recebido.

\section{REFERÊNCIAS}

ASSOCIAÇÃO BRASILEIRA DE NORMAS TÉCNICAS (ABNT). NBR-15575: Edificações habitacionais - Desempenho. Rio de Janeiro, 2013.

BRASIL. Ministério da Cidade. Regimento geral do Sistema Nacional de Avaliações Técnicas de produtos inovadores. Portaria MCid no 345. Brasilia, 2007.

Ministério da Cidade. Diretriz Sinat $\mathbf{n}^{\mathbf{0}}$ 005: Sistemas construtivos estruturados em peças de madeira maciça serrada, com fechamentos em chapas delgadas (Sistemas leves tipo "Light Wood Framing"). Secretaria Nacional da Habitação, Programa Brasileiro da Qualidade e Produtividade do Habitat (PBQP-H), Sistema Nacional de Avaliações Técnicas (SINAT): Brasília, setembro/2011.

. Ministério da Cidade. DATec $\mathbf{n}^{\circ} \mathbf{2 0}$ - Sistema construtivo TECVERDE: sistema leve em madeira. Secretaria Nacional da Habitação, Programa Brasileiro da Qualidade e Produtividade do Habitat (PBQP-H), Sistema Nacional de Avaliações Técnicas (SINAT): Brasília, outubro/ 2013.

BRASIL ENGENHARIA. Wood Frame recebe concessão da Caixa Econômica Federal visando o estímulo a novas tecnologias. Portal Engenho: São Paulo, julho/2013. Disponível em: <http://www.brasilengenharia.com/portal/construcao/5666-wood-frame-recebe-concessaoda-caixa-economica-federal-visando-o-estimulo-a-novas-tecnologias> Acesso em: 13 dez. 2013.

BUENO, C.M.D. Financiamento Construtivo (entrevista) in Téchne n. 136. PINI: São Paulo, Julho/2008.

BUETTNER, B. (relator). Projeto Tecnologia de Construção Sustentável. Relatório de planejamento estratégico. SENAI/PR: Curitiba, 2009. Disponível em: $<$ http://www.fiepr.org.br/para-

empresas/conselhos/base_florestal/uploadAddress/Relatorio[15473].pdf> Acesso em: 13 dez. 2013.

ELTZ, E.; SPEGGIORIN, E. Financiamento de casas de madeira pela Caixa Econômica Federal. Entrevista via correio eletrônico enviado em 07 nov. 2012. CEF - GIDUR/PO: Porto Alegre, 2012.

FERREIRA, R. MCMV de madeira in Construção, ed. 146. São Paulo: Pini, setembro/2013.

TÉCHNE. Pronta entrega. Téchne, n. 151. São Paulo: Pini, Outubro/2009. Disponível em: <http://techne.pini.com.br/engenharia-civil/151/artigo285472-1.aspx> Acesso em: $13 \mathrm{dez}$. 2013.

TECVERDE. Rede iVerde. Disponível em: <http://www.tecverde.com.br/site/> Acesso em: 13 dez. 2013. 